\title{
The Coupling of Stimulated Raman and Brillouin Scattering in a Plasma
}

\author{
D. Umstadter \\ W. B. Mori \\ C. Joshi
}

\section{DISCLAIMER}

This report was prepured as an account of work sponsored by an agency of the United States Government. Neither the United Stutes Government nor any agency thereof, nor uny of their employes, makes any warranty, express or implied, or assumes any legal liability or tesponsibility for the accuracy, completeness, or uselulnews of any information, appatalus, product, of process disclowe, or represints that its use would nol infringe privately owned rights. Refer. ence herein to any specifix commercial product, process, or service by Inade name, Irademark, genufacturer, or otherwise dos not nectsurily constitute or imply its endorsement, recom. mendation, or faverias by the United States Government or any apency therenf. The views and opinions of authors expresued berein do not vecessurily state or reflect those of the United Stata Goverameal of any ayeacy theroof.

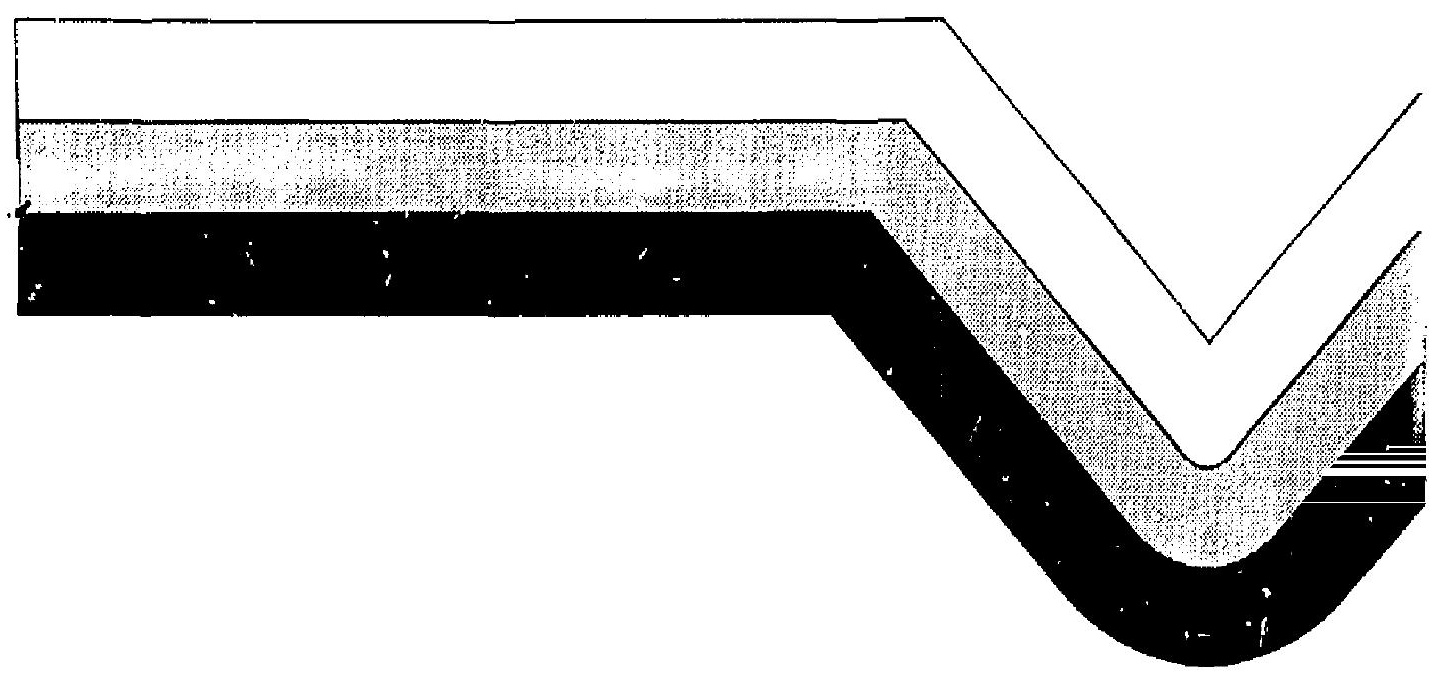


UCRL--21248

DE90 002825

\title{
The coupling of stimulated Raman and
}

\section{Brillouin scattering in a plasma}

D. Umstadter", W. B. Mori, and C. Joshi

University of California, Los Angeles

September 19,1988

\begin{abstract}
The obser ration of an anti-Stokes satellite in the spectrum of light backscattered from a $\mathrm{CO}_{2}$ laser plasme is reported. Its origin is found to be Thomson scattering of the incident light from a counterpropagating mode-coupled plasma wave. The parent electron and ion waves in the mode-coupling process were driven by stimulated Raman and Brillouin backscattering. The parent and daughter plasma waves were detected by ruby laser Thornsop scattering. A computer simulation modeling the experiment shows further cascading of the Stokes backscattered light to lower frequencies, apparently due to its rescattering by another, bigher phase velocity, counterprcpagating coupled mode. Comparisons with theoretical predictions are presented.
\end{abstract}

PACS numbers: $52.35 . \mathrm{Mw}$, 52.35.Fp, 52.40.Nk 


\section{Introduction}

Plasma wave mode coupling is a topic of current interest ${ }^{2}$ primarily because it may reduce the growth rate of stimulated Raman Scattering (SRS) and thereby increase the absorption of laser energy by laser-fusion plasmas. Specifically, mode coupling is predicted to trigger plasma wave collapse ${ }^{2}$. It is also used to explain the double peak in half-harmonic radiation ${ }^{3}$. Mode coupling occurs when an ion wave produced ripple modifies the electron plasma wave dispersion relation, allowing plasma-wrave spatial harmonics to be generated 4 . Coupled modes are observed in experiments in which the ion waves are driven by stimulated Brillouin scattering (SBS) and the electron plasma waves by either optical mixing ${ }^{5}$ or $\mathrm{SRS}^{6}$. Barr and $\mathrm{Chen}^{7}$ predict their amplitudes in the latter case.

A signature of mode coupling is an anti-Stokes satellite in the backscattered spectrum with a frequency equal to the sum of the incident light wave (pump) and the plasma wave frequency. It arises from Thomson scattering of the incident light wave from a counterpropagating coupled mode. We have observed the anti-Stokes satellite in the backscattered spectrum using an image dissector. Simultaneously, we have detected with ruby Thomson scattering a counterpropagating plasma wave with the same phase velocity as is predicted for the coupled mode. Another signature is a cascading of the backscattered light to lower frequencies, due to rescattering of the SRS backscattered light from another, higher phase velocity, coupled mode. We observed in a computer simulation not only the anti-Stokes satellite, but the cascading of the backscattered light to lower frequencies as well. The amplitudes of the coupled modes inferred from both the experiment and the simulation are 
consistent with the predictions of Bars and Chen ${ }^{7}$.

\section{Wave-Wave Interactions}

In order for SRS to accur, the three waves, the incident light wave $\left(\omega_{0}, k_{0}\right)$, the selecied light wave $\left(\omega_{1}, k_{1}\right)$ and the plasma wave $\left(\omega_{2}, k_{2}\right)$ must obey their dispersion relations, $\omega_{0,1}=\left(\omega_{p}{ }^{2}+c^{2} k_{0,1}{ }^{2}\right)^{1 / 2}$ and $\omega_{2}=\omega_{p}\left(1+3 k_{2}{ }^{2} \lambda_{D}{ }^{2}\right)^{1 / 2}$, and the phasematching conditions, $\omega_{0}=\omega_{1}+\omega_{2}$, and $k_{0}=k_{1}+k_{2}$, where $\lambda_{D}=\left(k_{B} T_{d} / m_{1} \omega_{p}^{2}\right)^{1 / 2}$ is the Debye length. If $k_{3} \lambda_{D} \ll 1$, then $\omega_{2} \simeq \omega_{p}$. In an underdense plasma $\left(\omega_{p} \ll \omega_{0}\right)$, these equations require that $k_{2} \equiv k_{1} \approx 2 k_{0}$ (Fig. 1(a)). In the case of SBS, the dispersion relation for electron waves is replaced with that for ion waves, $w_{2} \simeq k_{2} c_{2}$, where $c_{\mathrm{a}}=\left(\gamma Z k_{B} T_{c} / M\right)^{1 / 2}$ is the ion sound speed. Since $w_{z} \ll w_{0}$, phase-matching requires that $k_{2} \equiv k_{i} \simeq 2 k_{0}$ in this case as well (Fig. 1(b)).

Coupled modes have wavenumbers $k=k_{p}+m k_{i}$ and frequency $\omega \simeq \omega_{p}$, where $m$ is any positive or negative integer". When, in an underdense plasma, the ion wave is driven by SBS $\left(k_{i} \simeq 2 k_{0}\right)$ and the electron wave by SRS $\left(k_{\mathrm{p}}=2 k_{0}-\omega_{\mathrm{p}} / c \equiv 2 k_{0}-\delta k\right)$, the $m=0$ mode $\left.(\mid \omega, k]=\left[\omega_{p}, 2 k_{0}-\delta k\right]\right)$ has the same phase velocity and direction as an electron wave that would result from SRS backscattering in the absence of an ion ripple (Fig. $1(a))$. The $m=-1$ mode $\left([\omega, k]=\left[\omega_{p}, k_{p}-k_{i}\right] \simeq\left[\omega_{p},-\delta k\right]\right)$ has the same phase velocity $\left(v_{\phi} \simeq c\right.$ for $\left.\omega_{p} \ll \omega_{0}\right)$ as, but opposite direction to, an electron wave that would result from SRS forward scattering in the absence of an ion ripple $(F j g, 1(c))$. The $m=-2 \operatorname{mode}\left([\omega, k]=\left[\omega_{p}, k_{p}-2 k_{i}\right] \simeq\left[\omega_{p},-\left(2 k_{0}+\delta k\right)\right]\right\}$ has almost the same phast velocity as, but opposite direction to, the $m=0$ mode (Fig. 1(d)). The presence of the SBS induced density ripple, by generating these 
new modes, may reduce the growth rate of SRS'.

The incident $\mathrm{CO}_{2}$ pump light wave $\left(k=k_{0}\right)$ may Thomson scatter from the $m=-2$ mode $\left(k=-\left(2 k_{0}+6 k\right)\right)$, obeying the same phase-matching relations as above, to give backscattered light wave $\left(k=-\left[k_{0}+\delta k\right]\right)$ that is blue-shifted by $\omega_{\text {, }}$ (Fig: 1(e)). The SRS reflected light $\left(k=-\left|k_{0}-\delta k\right|\right)$ may Thomson scatter from the $m=-1$ mode $(k=-\delta k)$ to give a light wave $\left(k=-\left[k_{0}-2 \delta k\right]\right)$ that is propagating in the bacliward direction relative to the pump and that is red-shifted by $2 \omega_{p 1}$ a second Stokes satellite (Fig. 1(f)). The second Stokes satellite may also scatter from the $m=-1$ mode, and so on; in this way the backscattered light cascades to lower frequencies.

\section{Simulations}

A compuler simulation was performed in order to determine which modes should be expected in the experiment. The particle code that was used, WAVE ${ }^{8}$, is a fully relativistic electromagnetic particle-in-cell code. The following parameters were chosen in order to approximate the experiment: $k_{2} \lambda_{D}=0.2,(M / m)^{1 / 2}=100$, where $M / m$ is the ion to electron mass ratio, $\omega_{G} / \omega_{p}=3$ and $v_{0} / c=0.1$, where $v_{0}$ is the oscillating velocity of an electron in the field of the laser. Shown in the backscattered light frequency spectrum, $\left(E_{\text {trans. }}(\omega)\right)^{2}$, Fig. 2(a), are: the SBS signal at $\omega_{0}$, the first Stokes line due to scattering from the $m=0$ mode (SRS backscaiter), an anti-Stokes line due to scattering from the $m=-2$ mode, and a second Stokes line, due to rescattering of the first Stokes light from the $m=-1$ mode. Each line is separated in frequency by $\omega_{p}$. Fig, 2(a) represents the frequency spectrum at a 
time when SRS and SBS were occurring simultareously. Up to $100 \%$ of the incident light was seen to be Brillouin-backseattered in this simulation.

In order to make a better comparison with calculations of the mode-coupled wave amplitudes ${ }^{7}$, another simulation was performed with a fixed amplitude ion ripple $\left(\tilde{n}_{1} / n_{0}=20 \%\right)$ and immobile ions. In this simulation, SBS could not occur and the ripple amplitude was a constant. As shown in Fig. 2(b), which is the same as Fig. 2(a) except with a constant ion ripple, the anti-Stokes satellite was again seen in the baclsscattered spectrum. However, two differences between this result and that of the previous simulation were observed: the SRS was reduced considerably, and the second Stokes satellite was not observed. The reduction of SRS in the fixed ion ripple case arises from the fact that, from the start of the simulation, a large fraction of the pump light was reflected by the ripple and was thus unavailable to Raman scatter. The assertion that the second Stokes satellite is due to rescattering of the SRS backscatter explains why no second Stokes satellite was observed in the simulation with reduced SRS backscatter.

\section{Experiments}

The experimental arrangement was similar to the one discussed previously ${ }^{9}$. The plasma waves were excited with light from a $10.6 \mu \mathrm{m} \mathrm{CO} 2$ laser ( $10 \mathrm{~J}, 2 \mathrm{~ns}$ ), focussed by an $f / 7.5$ lens to a spot of diameter $d \leq \hat{0} 00 \mu \mathrm{m}$ onto an arc preionized $\mathrm{H}_{2}$ plasma. The backscattered light was diverted from the path of the incident (pump) $\mathrm{CO}_{2}$ beam by a beamsplitter. Its amplitude could then be analyzed as a function of time with a resolution of $250 \mathrm{ps}$ using a liquid He cooled detector in combination with a 
grating spectrograph, or as a function of frequency by adding an image dissector ${ }^{10}$. The plasma waves were directly detected by Thomeon ccattering using a $1 \mathrm{~J}, 15$ ns ruby laser pulse $(\lambda=.6943 \mu \mathrm{m})$, which was oynehronized in time with the $\mathrm{CO}_{2}$ pulse and brought transversely to 2 focus at the location of the $\mathrm{CO}_{2}$ beam waist. The amplitude of the reimaged Thomson-scattered light was analyzed as a function of frequency by using an optical multichannel analyzer (OMA) in tandem with a grating spectrograph. The freguency of the plasma wave was determined by the frequency shift of either the SRS or the Thomson scatter. Its direction relative to the pump was determined by the sign of the frequency shift. Its wavenumber was determined by adjusting the scattering angle $\left(\theta_{1}\right)$ between the incident beam and the collection lens for the Thomson-scattered beam. This angle is uniquely determined by the wavenumber of the plasma wave, $k_{2}$, using the $k$-matching condition, $\mathbf{k}_{\text {prob }}=$ $\mathbf{k}_{1} \pm \mathbf{k}_{2}$, where $k_{\text {probe }}$ and $k_{1}$ are the wavenumbers of the ruby probe and scattered beam, respectively.

Shown in an oscilloscope trace of the image dissector output, Fig. 3, are two backscattered satellites, a red-shifted satellite on the right, resulting from the $\mathrm{CO}_{2}$ laser scattering from the $m=0$ mode (SRS), and a blue-shifted satellite on the left, resulting from the pump Thomson scattering from the $m=-2$ mode; both are shifted from the incident pump wavelength by the same amount. The unshifted line that results from SBS is not seen in Fig. 3 because it was absorbed with an absorber $\left(\mathrm{SF}_{6}\right)$ in order to prevent saturation of the detector. The two signals in Fig. 3 were attenuated by different amounts, and so the ratio of their amplitudes in Fig. 3 does not reflect the actual ratio.

Stokes and anti-Stokes satellite pairs are reported by Batha, et al. ${ }^{\text {.l }}$ but their 
origin is attributed to enhanced Thomson scattering from hot electrons that originated at the critical layer. In the present experiment, the SRS frequency shift uniquely determined the density to be $2.0 \times 10^{16} \mathrm{~cm}^{-3}$, much less than the critical density $\left(n_{0} / n_{c}=2 \times 10^{-3}\right)$, and estimates of the density profile indicate that no critical layer existed. Furthermore, no electrons were detected over a range of 6 to $100 \mathrm{KeV}$ and with a sensitivity of $10^{5}$ electrons/steradian; KeV. The anti-Stokes satellite was observed in the simulations even though a homogeneous density profile was used, and so, of course, no critical layer could have existed. For these reasons, the explanation of Batha, et al. cannot be applicable to the results of either our experiments or simulations. On the other hand, mode coupling may be re'evant to their results since it may produce anti-Stokes sidebands all the way up to $n_{\odot} / 4$, provided SRS and SBS coincide with each other in both space and time.

Simultaneously with the results of Fig. 3, three narrow lines in the frequency spectrum were observed with ruby Thomson scattering when the collection lens for the ruby Thomson-scattered ligit was at $\theta_{\mathbf{J}}=\mathbf{7}^{\circ}$, the angle corresponding to $k=$ $2 k_{0}(1 \pm 15 \%) ;$ the uncertainty was due to the $4.5^{\circ}$ angle subtended by the collection lens. This is the wavenumber of each of the following waves: the SBS-induced ion wave, the $m=0$ mode, and the $m=-2$ mode. The peak in the center of Fig. 4, which shows a photograph of the optical multichannel analyzer output, is due to Thomson scattering from an SBS induced ion wave. The Thomson-scattered signal from the ion wave appears unshifted in frequency because the ion-acoustic frequency, $w_{i}$, was less than the resolution of the spectrometer. The acoustic frequency was, nevertheless, measured by using a spectrometer with a grealer resolution, and in this way the electron temperature was determined to be $T_{6} \simeq 10 \mathrm{eV}$. The peak on 
the right of Fig. 1 is due to Thomson-scattered light that is red-shifted in frequency $(\Delta f)$ by an amount that, assuming $\Delta \omega=\omega_{p}$ and $T_{c}=10 \mathrm{eV}$, is consistent with a plasma density of $2.0 \times 10^{16} \mathrm{~cm}^{-3}\left(n_{0} / n_{\mathrm{c}}=2 \times 10^{-3}\right)$ and a value $k_{2} \lambda_{D}=0.2$. This feature was detected only when the $\mathrm{CO}_{2}$ laser was incident on the plasma and thus was not due to ruby stray light entering the spectrometer. The result $k \lambda_{D} \ll 1$ implies that this was collective scattering. By varying the arc fill pressure and the relative timing between the preionization and the laser pulse, the plasma density could be continuously adjusted. The densities calculated from the frequency shifts of both the red-shifted SRS (Fig. 3) and the red-shifted peak of the $7^{\circ}$ ruby Thomson scattering agree within the experimental error over the density range of the experiment. The red-shifted peak in Fig. 4, therefore, appears to be due to scattering from the $m=0$ mode. The peak on the left of Fig. 4 is a blue-shifted satellite, which is the counterpart of the blue-shifted backscattered satellite shown in Fig. 3. It was shifted by the same frequency as, and was detected only in the presence of, the red-shifted line. Its direction and phase velocity are signatures of the $m=-2$ mode. The amplitudes shown in Fig. 4 do not represent the actual relative amplitudes because different frequencies were attenuated by different amounts.

\section{Discussion and Summary}

The Bragg scattering formula ${ }^{12}$ relates $\tilde{n}$, the perturbed electron density, to the scattered light power $\left(P_{1}\right)$ normalized to the incident power $\left(P_{0}\right)$ and applies to 
both Thomson scattering and SRS in the limit of $\tilde{n}_{1} / n_{0} \ll 1$ :

$$
\frac{E_{0} / r_{p}}{E_{0} / r_{0}} \simeq \frac{P_{0}}{P_{0}}=\frac{1}{4} \tilde{n}^{2} r_{0}^{2} \lambda_{\text {probe }}^{2} d^{2}
$$

The variable to is the classical electron radius, $d$ is the width of the interaction region, and $r_{p} / r_{0}$ is the ratio of the ruby pulse length to the lifetime of the plasma wave. We may use the Bragg formula to determine $\bar{n}$ of the various coupled modes from the amount of light that they scatter and compare the results with the amplitudes predicted by Barr and Chen ${ }^{7}$.

Mode coupling theory predicts that the ratio of the perturbed density of the $m=-2$ mode to that of the $m=0$ mode should be proportional to the size of the ion ripple. This prediction is in qualitative agreement with the relative amount of light scattered from these modes in the experiment for various amplitudes of the ion ripple. Specifically, the amplitude of the blue-shifted feature of the ruby Thomson-scattered spectrum (Fig. 4) appeared to be correlated with the product of the amplitudes of the red-shifted satellite and the line due to scattering from the ion wave ${ }^{13}$. The absolute amplitudes agree with theory as well. In particular, Barr and $\mathrm{Chen}^{7}$ predict that this ratio of the perturbed density of $m=-2$ mode to that of the $m=0$ mode may be $2 \%-10 \%$ for an average ion ripple of $3 \%-10 \%$. When the actual attenuation factors are taken into consideration, this prediction is found to be consistent with the ratio that was measured by both ruby Thomson scattering and backscattering (approximately $4 \%$ ) for the same average value for the ion ripple amplitude as above, inferred from the SBS. It is also predicted ${ }^{7,11}$ that this ratio should be approximately unity for a ripple c. $20 \%$. A ratio of unity may indeed be inferred from the annount of light scattered in the simulation, (Fig. 2(b)), where the 
ripple amplitude was also $20 \%$.

None of the modes with valies $m>0$ or $m<-2$ were observed in the present experiment. This could be because spatial harmonics, with greater values of $k$, but comparable values of $w$, are more heavily Landau damped, since Landau damping increases with $v_{e} / v_{q}=v_{c} k / \omega$. From the relation $v_{d} / v_{\phi}=|m+1| k_{2} \lambda_{D}$, we see that, at the density typically used in the experiment $\left(k_{2} \lambda_{D}=.2\right)$, the values $m>0$ and $m<-2$ correspond to $v_{d} / v_{t} \geq 4$, as compared to $v_{d} / v_{d}=.2$ for $m=0$. This explanation is supported by the fact that we never observed the $m=0$ mode when its value for $v_{\mathrm{c}} / v_{\mathrm{d}}$ was doubled to .4 , which was obtained by decrezaing the plasma. density by a factor of four, and thereby decreasing the plasma wave frequency $\left(\omega=\omega_{p}\right)$ by a factor of two, but leaving $k$ unchanged. Other authors also gredict that the larger-k modes would be lower in amplitude. Specifically, the waves with $k$ equal to $k= \pm 4 k_{0}$ are predicted ${ }^{7}$ to have amplitudes that are $20 \%$ of the amplitudes of the waves with $k= \pm 2 k_{0}$. We would therefore not expect to see the $k \geq 4 k_{0}$ modes since their amplitudes would have been beneath the detection threshold in the experiment.

As is shown in Fig. 1(g), there is another way in which the blue-shifted satellite may arise. The SBS reflected light $\left(k \simeq-k_{0}\right)$ may Thomson scatter from the $m=-1$ mode $(k=-\delta k)$ and result in a light wave propagiting in the backscattered direction $\left(k=-\left|k_{0}+\delta k\right|\right)$ that is blue-shifted by $\omega_{p}$. This contribution to the blue-shifted satellite should be less than the contribution due to the pump Thomson scattering from the $m=-2$ mode, which is shown in Fig. 1(e), by the product of SBS reflectivity and the square of the ratio of the dessity perturbation of the fast $(m=-1)$ to the slow ( $m=-2)$ counterpropagating waves, 


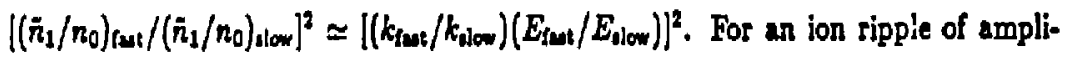
tude from 3-18\%, as in our experiment, it is predicted ${ }^{7,15}$ that $E_{\text {Iuc }} / E_{\text {dow }}$, the satio of the electric field amplitude of the fast $(m=-1)$ wave to that of the slow $(m=-2)$ wave, varies from unity by less than $50 \%$ [sce their Fig. 3]. Thus

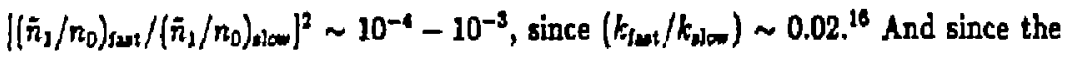
SBS relectivity was $\leq 10 \%$, Thomson scattering of the SBS reflected light should be negl:gible compared to Thomson scattering of the pump, ie. less by a factor of $10^{-5}-10^{-4}$.

An electron wave with the same phase velocity as the $m=-1$ mode $([\omega, k]=$ $\left.\left[\omega_{p}, k_{0}-k_{1}\right]=\left[\omega_{p},-\delta k\right]\right)$ may arise from a different mechanism, collinear optical mixing of the SRS reflected light $\left(k=-\left[k_{0}-\delta k\right]\right)$ with the SBS refected light $\left(k=-k_{0}\right)$ (Fig. 1(h)). Therefore, as described in the previous paragraph, the SBS rellected light is properly $k$-matched to Thomson scatter from the resulting fast wave to give a blue-shifted satellite $\left(k=-\left[k_{0}+\delta k\right]\right)$ (Fig. 1(g)). In c.ptical mixing, two light waves, whose frequency difference is equal to the Bohm-Gross frequency, beat to excite a plasma wave. A comparison of the growth rates shows that optical mixing is less effective than mode coupling in the regime of our experiment in exciting the fast wave. ${ }^{13}$ Even if we suppose optical mixing and mode coupling were of the same order, the contribution to the blue-shifted satellite due to Thomson scattering of the SBS reflected light from the beat wave would be less than that due to Thomson scattering of the pump from the $m=-2$ mode by a factor of $\leq 10 \%$, the SBS reflectivity.

There are several mechanisms other than mode coupling that could also possibly give tise to a counterpropagating slow wave $\left(\omega_{p}, k \simeq 2 k_{0}\right)$. For instance, a 
counterpropagating slow wave could conceivably result from the sefiection of the copropagating slow wave from its critical layer, if one exists. It could also result if the SBS beckscatter were to Raman backscatter. Finally, such a wave may be excited by the electron decay instability in which a copropagating electron wave generates an ion wave with $k=4 k_{0}-2 \delta k$ and is reflected (Fig. 1(i)). Even if they were not the result of higher order processes ${ }^{13}$, the waves that would arise fr.mm any of these competing mechanisms would not give a blue satellite. This is because, as was shown in Fig. 1(e), in order for a wave to $k$-match to the pump and a backscattered light wave that is blue-shifted by $\omega_{p}$, it would have to have wa"enumber $k \simeq-\left(2 k_{0}+\delta k\right)$, rather than $-\left(2 k_{0}-c k\right) . \triangle$ similar argument may be applied to ruby Thomson scattering. The waves that would be generated by any of the competing mechanisms would have $k \simeq-\left(2 k_{0}-6 k\right)$. Therefore, of the mechanisms that could drive slow counterpropagating waves, only mode coupling can explain the observed ruby and $\mathrm{CO}_{2}$ Thomson seattered spectra.

In summary, an anti-Stokes satelite in the backscattered spectrum appears to originate from Thomson scattering of the pump lignt from a coupled mode ( $m=-2$ ) that arose from the coupling of SRS and SBS induced electron and ion waves. The amplitude of the $m=0$ mode, relative to the $m=-2$ mode, inferred from either the ruby probe or the backscatter, supports the model of Barr and Chen ${ }^{7}$. The same appears to hold true for different values of the ion ripple. $\Lambda$ cascading to lower frequency observed in the simulation is consistent with rescattering of the SRS backscattered light from the $m=-1$ mode. Finally, since the siow counterpropagating waves that could be excited by any competing mechanisms are not phase-matched, only mode coupling can explain the observed scattered spectra. 


\section{Acknowledgments}

We thank Professor F. F. Chen and Dr. C. Clayton for many useful discussions.

This work was supported by the U.S. Department of Energy DE-AS03-83-ER-40120, NSF Grant ECS 83-10972 and the LLNL University Research Program. 


\section{References}

[a] Present address: AT\&T Bell Laboratories, Murray Hill, NJ 07974.

[1] D. M. Villeneuve, H. A. Baldis and J. E. Bernard, Phys. Rev. Lett. 59, 1585 (1987); C. J. Walsh, D. M. Villeneuve, and H. A. Baldis, Phys. Rev. Lett. 53, 1445 (1984).

[2] C. H. Aldrich, B. Bezzerides, P. F. Du Bois, and H. A. Rose, Comments Plasma Phys. Controlled Fusion 10, 1 (1986); H. A. Rose, D. F. DuBois, and B. Bezzerides, Phys. Rev. Lett. 58. 2547 (1987); W. Rozmus, R. P. Sharma, J. C. Simon, and W. Tighe, Phys. Fluids 30, 2181 (1987).

[3] H. Figueroa and C. Joshi, Phys. Fluids 30, 2294 (1987).

[4] P. K. Kaw and J. Dawson, Phys. Fluids 16, 1967 (1973).

[5] C. Darrow, D. Umstadter, T. Katsouleas, W. B. Mori, C. E. Clayton, and C. Joshi, Phys. Rev. Lett. 56, 2629 (1986).

[6] D. M. Villeneuve, C. J. Walsh, and H. A. Baldis, Phys. Fluids 28, 1591 (1985).

[7] H. Barr and F. Chen, Phys. Fluids 30, 1180 (1987).

[8] R. L. Morse and C. W. Neilson, Phys. Fluids 14, 830 (1971).

[9] D. Umstadter, R. Williams, C. Clayton and C. Joshi, Phys. Rev. Lett., 59, 292 (1987).

[10] H. A. Baldis, N. H. Burnett, and M. C. Richardson, Rev. Sci. Instrum. 48, 173 (1977). 
[11] S. H. Batha, R. Bahr, L. M. Goldman, W. Seka, and A. Simon, Bull. Am. Phys. Suc. 32, 1715 (1987).

(12) R. E. Slusher and C. M. Surko, Phys. Fluids 23, 472 (1980).

[13] D. Unstadter, Ph. D. thesis, Univ. of Calif., Los Angeles (1987).

[14] From the relative growth rates of the various predicted modes ${ }^{7}$, only the fastest growing mode need be considered, Barr and Chen's $\omega_{1}$ mode /see their Fig. 1(b), using $g=(\epsilon / 6)\left(c^{2} / v_{c}^{2}\right)\left(n / n_{c}\right)=3.4$ where $\epsilon$ is the ion ripple density amplitude normalized to the background density and the other variables are as defined previously].

[15] Using $q=(\epsilon / 6)\left(c^{2} / v_{\mathrm{f}}^{2}\right)\left(n / n_{\mathrm{f}}\right)=0.5-3.0$.

[16] Again for this range of values for $q$, only the fastest growing mode, Barr and Chen's $\omega_{1}$ mode, need be considered [see their Fig. 1(b)]. 


\section{Figure Captions}

Figure 1. Wavenumber matching diagrams for: A) fundamental scattering processes $(a, b) ; B)$ mode-coupling processes $(e-f)$; C) alternative mechanisms $(g-i)$. Solid curves: electrostatic waves; open arrows: light waves; striped arrows: idler or scattering waves. In each diagram, the initiating wave is at the uyper left. (a) SRS, or production of the Stokes satellite from the $m=0$ mode. (b) SBS. (c) Prcduction of the $m=-1$ coupled mode. (d) Production the $m=-2$ mode. (e) Seattering of the pump light from the $m=-2$ mode to give the blue satellite. (f) Scattering of the Stokes satellite from the $m=-1$ mode to give the second Stokes satellite. ( $\mathrm{g}$ ) Alternative production of the blue satellite by scattering of the SBS-reflected light from the $m=-1$ mode. (h) Alternative production of the $m=-1$ mode by optical mixing of backscattered wares. (i) Alternative production of a baçkward slow wave by electron decay of forward wave into a backward wave and an ion wave.

Figure 2. Infrared backscattered frequency spectra from computer simulations: The horizontal axes are in units of frequency, normalized to the plasma frequancy; $\omega / \omega_{\mathrm{p}}=3$ corresponds to $\omega_{0}$, the incident light frequency. (a) Results from a computer simulation that models the experiment. The peaks from right to left are the resull of scattering from: the $m=-2$ mode, the ion wave, the $m=0$ mode, the $m=-1$ mode. (b) Results from a simulation with a constant ion ripple (20\%) and immobile ions. The peaks are the same as in (a) except for a reduced SRS amplitude and the absence of a second Stokes satellite.

Figure 3. Experimentally measured frequency spectrum of the infrared backscat- 
tered light: the $m=0$ mode (right) and the $m=-2$ mode (leit).

Figure 4. Frequency spectrum of the ruby Thomson-scattered light: the $m=0$ coupled mode (tight), the ion wave (center) and the $m=-2$ coupled mode (left). 


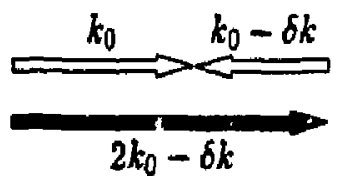

(a)

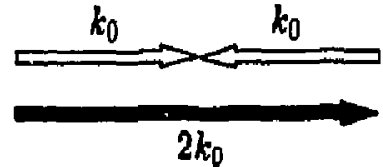

(b)

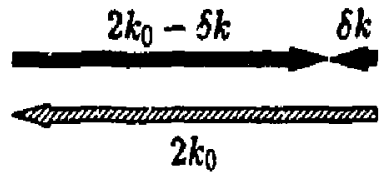

(c)

$$
2 k_{0}-6 k
$$

$2 k_{0}+\delta k$

Q2

(d)

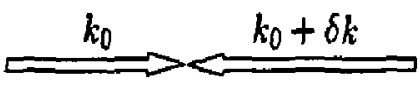

प) $2 k_{0}+\delta k$

(e)

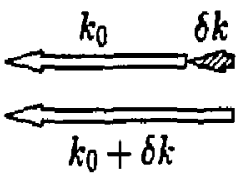

(g)

$$
\sum_{\delta k k_{0}-2 \delta k}^{k_{0}-\delta k}
$$

(f)

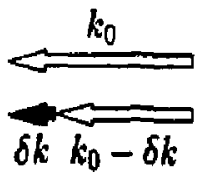

(h)

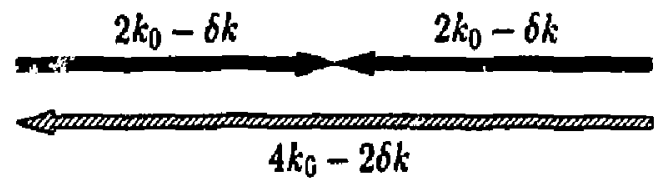

(i) 


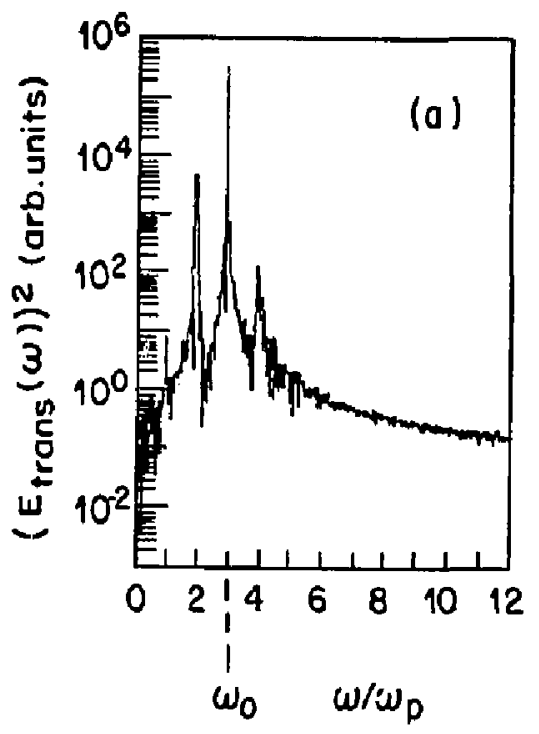

Figure 2 (a) 


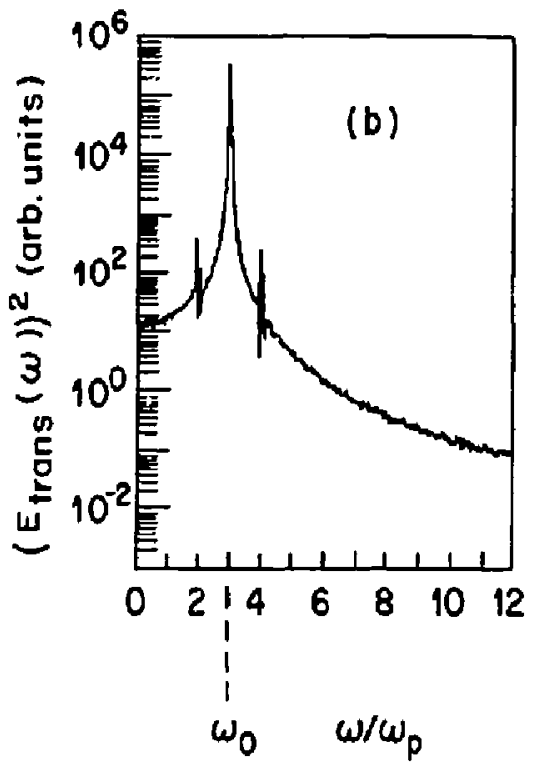

F1gure 2 (b) 


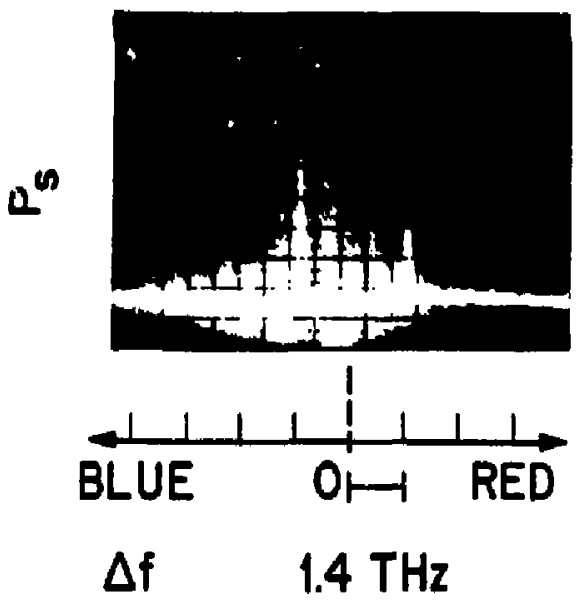

Figure 3 


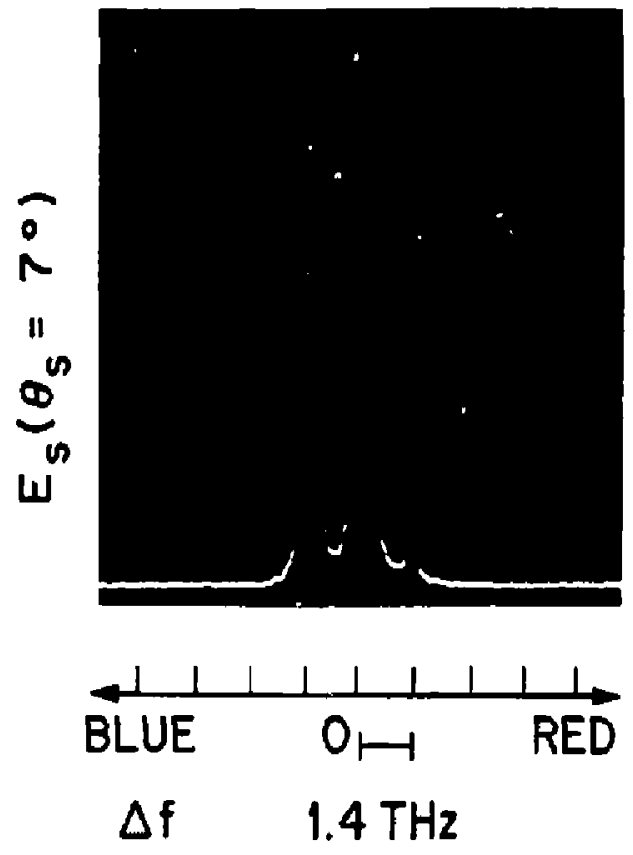

Figure 4 